

\title{
Diagnóstico y Cuantificación de Pérdidas en la Gestión Logística de Almacenamiento en la Comercialización de Productos Pesqueros
}

\author{
Alain Ulloa Zaila ${ }^{1^{*}(D)}$, Damaris Taydi Castillo Jiménez ${ }^{1}$ (D), Dariel Rivadeneira \\ Casanueva $^{1}$, Orlando de la Cruz Rivadeneira ${ }^{2}$, Elonay Lizandy Mederos Yumar $^{1}$ \\ ${ }^{1}$ Departamento de Ingeniería Industrial, Facultad de Ciencias Técnicas y Empresariales, \\ Universidad de Sancti Spíritus “José Martí Pérez", C.P. 60100, Cuba. \\ ${ }^{2}$ Empresa Pesquera de Sancti Spíritus "PESCASPIR”, C.P. 60100, Cuba. \\ *Autor de correspondencia: alainu@ uniss.edu.cu \\ https://doi.org/10.22209/rt.v44n3a05 \\ Recepción: 01 de diciembre de 2020 | Aceptación: 14 de junio de 2021 | Publicación: 01 de agosto 2021
}

\section{Resumen}

La investigación se realizó en la unidad comercializadora de la empresa pesquera de Sancti Spíritus donde se identificaron limitaciones en la gestión de almacenamiento. Para resolver la problemática identificada, se planteó como objetivo implementar un procedimiento para el diagnóstico y cuantificación de pérdidas en la gestión logística de almacenamiento. El procedimiento diseñado contiene un conjunto de fases en las que se desarrollan diferentes métodos y técnicas como el método Delphi, lista de verificación, diagrama de flujo de proceso, análisis modal de fallos y efectos, gráficos de control, histograma y la función de pérdida de Taguchi. Como resultados se obtuvieron: los fallos que ocurren dentro de la cámara de frío con sus niveles de prioridad de riesgo; se determinó que la variable temperatura estaba fuera de los parámetros de control establecidos; se calculó la capacidad del proceso en la cámara de frío y se cuantificaron pérdidas económicas relacionadas con las ineficiencias en el almacenamiento. La investigación permitió detectar las principales causas que influyen en la improductiva gestión logística de almacenamiento de la organización, las pérdidas asociadas y se propusieron medidas para la solución de los problemas identificados.

Palabras clave: calidad; cuantificación de pérdidas; gestión logística; logística de almacenamiento; mejora continua.

\section{Diagnosis and Quantification of Losses in the Logistical Management of Storage in the Commercialization of Fishery Products}

\begin{abstract}
The research was carried out in the commercialization unit of the fishing company of Sancti Spíritus where limitations in storage management were identified. To solve the identified problem, the objective was to implement a procedure for the diagnosis and quantification of losses in storage logistics management. The designed procedure contains a set of phases in which different methods and techniques are developed, such as the Delphi method, checklist, process flow diagram, modal analysis of failures and effects, control charts, histogram and Taguchi's loss function. As results, the following were obtained: the failures occurring inside the cold chamber with their risk priority levels; the temperature variable was determined to be outside the established control parameters; the capacity of the process in the cold chamber was calculated; and economic losses related to inefficiencies in storage were quantified. The research made it possible to detect the main causes that influence the unproductive storage logistics management of the organization, the associated losses and proposed measures for the solution of the problems identified.
\end{abstract}

Keywords: continuous improvement; logistic administration; loss quantification; storage logistic; quality. 


\section{Introducción}

La cuestión de las pérdidas de alimentos es muy importante en los esfuerzos para combatir el hambre, aumentar los ingresos y mejorar la seguridad alimentaria en los países más subdesarrollados del mundo. Dicha cuestión afecta a la seguridad alimentaria de los pobres, a la calidad y la inocuidad alimentaria, al desarrollo económico y al medio ambiente (FAO, 2012). Las causas exactas de las pérdidas de alimentos varían en las diferentes partes del mundo y dependen sobre todo de las condiciones específicas y situación local de cada país. Los rangos de cantidades de pérdida en la post cosecha son del 20 al 60 por ciento de la producción total por países y aproximadamente un tercio de la comida producida para el consumo humano se pierden o desperdician globalmente en cantidades aproximadas a 1,3 mil millones de toneladas por año. Las causas de pérdidas son las ineficiencias en las etapas de producción, post cosecha, almacenamiento, manejo, y transporte (Lemma et al., 2014; Gao et al., 2017; Duan y Liu, 2019).

Día a día, las cadenas de suministro (CS) son más complejas pues se extienden alrededor del mundo e involucran más productos y participantes. Los ejecutivos a cargo de la cadena deben tratar con clientes exigentes, mercados que cambian a gran velocidad y tienen costos más altos (Kaufmann y Gaeckler, 2015). Las CS o de abastecimiento se describen como los recursos interconectados y las actividades necesarias para crear y entregar productos y servicios a los clientes, por lo cual se extienden desde el punto donde se extraen los recursos naturales hasta el consumidor (Sánchez y Hasbleidy, 2014). El subsistema de gestión de almacén, dentro de una cadena de suministro, es un punto en el que confluyen intereses de diferentes departamentos de la empresa, los cuales necesitan de un adecuado funcionamiento del mismo para poder cumplir con sus objetivos (Koldborg et al., 2013). Este subsistema, del sistema logístico de distribución, se ha convertido en uno de los más importantes, consecuencia de su incidencia en el servicio al cliente y en los costes operativos de la empresa (Pulido, 2014). La mayoría de los alimentos perecederos son sensibles a la temperatura y esta es la principal condición ambiental que resulta en la degradación de la calidad. Por lo tanto, el control de la temperatura es esencial para garantizar la calidad y seguridad de los alimentos perecederos (Xue et al., 2014).

Cuba se encuentra inmersa en un proceso de transformaciones de su economía, para sentar las bases del desarrollo económico que permita perfeccionar su sistema social socialista (Castillo-Jiménez et al., 2021). En medio de este proceso, la acuicultura ha desarrollado y fomentado en Cuba la cultura tecnológica que conlleva todos los sistemas de cultivo de peces de agua dulce, donde el último eslabón de esta cadena es el procesamiento industrial de las producciones logradas, prestándose gran interés a la inocuidad del producto final y sus derivados (Galindo-López et al., 2009).

La empresa pesquera de Sancti Spíritus, tiene como misión cultivar de forma extensiva e intensiva especies acuícolas para su procesamiento industrial. Su funcionamiento económico está establecido a partir de los procesos claves: cultivo, reproducción de alevines, industrialización y comercialización. La empresa presenta como deficiencias en el sistema logístico de distribución, inadecuada manipulación del producto terminado, obsolescencia en la tecnología de almacenamiento, desconocimiento en la metodología de almacenamiento, desconocimiento de la cuantía de las pérdidas en los subsistemas logísticos de almacén. A partir de dicha situación se define como problema a resolver que, las limitaciones en la gestión logística de almacenamiento en la industria pesquera de Sancti Spíritus, imposibilita conocerlas pérdidas de productos terminados. El objetivo de la investigación consistió en implementar un procedimiento para el diagnóstico de la gestión logística de almacenamiento en la industria pesquera de Sancti Spíritus, que permita cuantificar las pérdidas de productos terminados.

\section{Materiales y Métodos}

El procedimiento diseñado, que se muestra en la Figura1, permitió diagnosticar y cuantificar pérdidas en la gestión logística de almacenamiento; específicamente en la cámara de frío, donde se almacenan los productos terminados. Esto fue posible mediante la aplicación de herramientas como la lista de verificación, el análisis modal de fallos y efectos (AMFE), la evaluación de la capacidad del proceso con gráficos de control e histograma y la función de pérdida de Taguchi (Gutiérrez-Pulido y de la Vara-Salazar, 2009). 


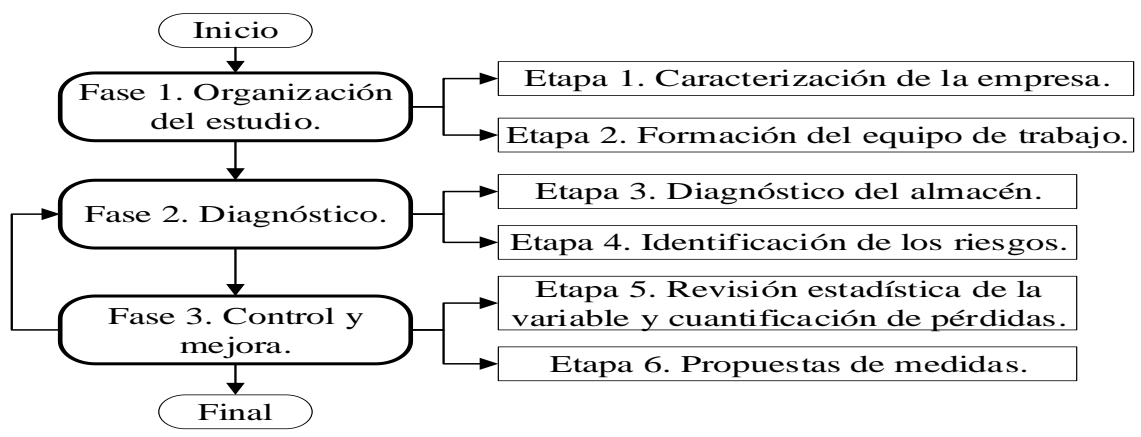

Figura 1. Procedimiento para el diagnóstico y la cuantificación de pérdidas en la gestión logística de almacenamiento.

\section{Fase 1: organización del estudio}

En esta fase se procedió a realizar un análisis de la organización objeto de estudio y sus almacenes, donde se tiene en cuenta las etapas que se muestran en la estructura del procedimiento detallado en la Figura 1, las cuales se describen a continuación.

Etapa 1, caracterización de la empresa: la caracterización de la empresa es fundamental para tener conocimiento de forma general de la organización y de aquellos elementos que le permiten identificarse del resto de las entidades.

Etapa 2, formación del equipo de trabajo y descripción de los procesos que ocurren en el almacén: en esta etapa se utilizó el método de selección de expertos, aprobado en la NC 49:1981 (ONN, 1981). Para su desarrollo se aplicó una encuesta que permitió realizar un análisis de los candidatos mediante la determinación del coeficiente de competencia de los mismos. Luego, mediante la Ecuación (1), se calculó la cantidad de expertos necesarios para la investigación (ONN, 1981):

$$
M=\frac{p(1-p) k}{i^{2}}
$$

Donde: M: cantidad de expertos, i: nivel de precisión deseado, p: proporción estimada de errores de los expertos, k: constante, cuyo valor está asociado al nivel de confianza elegido.

Luego, se realizó una descripción detallada de los procesos que ocurren en el almacén, lo cual permite familiarizarse con las actividades específicas que en cada una de sus partes acontece. Para la realización de este paso se utilizó la herramienta diagrama de proceso, la cual es una representación gráfica de la secuencia de los pasos o actividades de un proceso, que incluye transportes, inspecciones, esperas, almacenamientos y actividades de retrabado o reproceso (Gutiérrez-Pulido y de la Vara-Salazar, 2009).

\section{Fase 2: diagnóstico}

En esta fase se procedió a realizar un diagnóstico del subsistema gestión de almacén, para detectar las deficiencias, evaluar cómo estas influyen en las pérdidas, y, posteriormente, proponer mejoras a los problemas detectados en el diagnóstico. A continuación, se describen las etapas que conforman la presente fase.

Etapa 1, diagnóstico del almacén: esta etapa de trabajo constituye el inicio del diagnóstico que se realiza al almacén y abarca el estudio de la instalación física y su gestión preferiblemente de manera cualitativa y cuantitativa. Para el desarrollo de este paso se utilizó la herramienta de lista de verificación; que permite, tener en cuenta las normas, resoluciones y requisitos, para observar si existen o no dichos requisitos y condiciones; y así diagnosticar la situación actual del subsistema objeto de estudio en la investigación.

Etapa 2, identificación de los riesgos: en esta etapa se analizó los resultados arrojados por la lista de verificación en el diagnóstico. Después de comprobar los problemas existentes, se determinaron las posibles causas que los 
ocasionan y se procedió a identificar los riesgos de estos mediante la herramienta de AMFE, para describir de forma estructurada las relaciones de funcionamiento y los posibles errores, y se determinan los niveles de prioridad de riesgo (NPR), a partir de los cuales se obtiene información sobre la urgencia de los posibles riesgos, y la búsqueda de acciones de mejora (James, 1997). Se pueden distinguir dos tipos de AMFE: el AMFE de producto/servicio y el AMFE de proceso. El destinado hacia el producto/servicio sirve como herramienta de optimización para su diseño. Consiste en el análisis preventivo de los diseños y en buscar anticiparse a los problemas y necesidades de los mismos. Este AMFE es el paso lógico previo al de proceso porque se tiende a mejorar el diseño, para evitar el fallo posterior en producción. En el AMFE de proceso se analizan los posibles fallos que pueden ocurrir en los diferentes elementos del proceso (materiales, equipo, mano de obra, métodos y entorno), y cómo éstos influyen en el producto resultante (Gutiérrez-Pulido y de la Vara-Salazar, 2009).

\section{Fase 3: control y mejora}

En esta fase se controló estadísticamente la variable, se cuantificó pérdidas y se propusieron medidas a los problemas detectados en el diagnóstico. A continuación, se describen las etapas que conforman la presente fase.

Etapa 1, revisión estadística de las variables y cuantificación de pérdidas: en esta etapa se determinó y evaluó las variables resultado, es decir, la de mayores perturbaciones o los de mayores NPR del subsistema de almacenamiento, obtenido en el paso anterior con la aplicación del AMFE y se comprobó si el proceso de almacenamiento esta en control estadístico, mediante las cartas o gráficos de control, y si el proceso es capaz. Posteriormente, se cuantificó las pérdidas que ocasionan estas perturbaciones.

Paso 1, cartas o gráficos de control: existen dos tipos generales de cartas de control: para variables y para atributos. Las cartas de control para variables se aplican a características de calidad de tipo continuo, que intuitivamente son aquellas que requieren un instrumento de medición (peso, volumen, voltaje, longitud, resistencia, temperatura y humedad) (Gutiérrez-Pulido y de la Vara-Salazar, 2009). En la investigación, para el análisis del estado de control estadístico del subsistema de almacenamiento, se utilizaron los gráficos de control por variables de valores individuales $(\overline{\boldsymbol{X}})$ y recorridos $(R)$.

Paso 2, evaluación de la capacidad del proceso: la capacidad del proceso se define como los límites de la variabilidad dentro de los cuales el proceso opera, mientras las circunstancias existentes en ese momento se mantengan (Gutiérrez-Pulido y de la Vara-Salazar, 2009). Una vez lograda la estabilidad del proceso, se realiza el cálculo de índice de capacidad (Cp, proceso centrado; $\mathrm{Cpk}$, proceso no centrado), según la Tabla 1.

Tabla 1. Expresiones de cálculo para indicadores de capacidad del proceso de almacenamiento (Gutiérrez-Pulido y de la Vara-Salazar, 2009).

\begin{tabular}{cclc}
\hline $\begin{array}{c}\text { Proceso centrado en su valor } \\
\text { nominal }\end{array}$ & $\begin{array}{c}\text { Proceso no centrado en su } \\
\text { valor nominal }\end{array}$ & Limites mínimo de evaluación \\
\hline $\mathrm{Cp}=\frac{\mathrm{LSE}-\mathrm{LIE}}{6 \sigma}$ & $\mathrm{Cpk}=\operatorname{mínimo}\left(\frac{\mathrm{S} 1}{3 \sigma}, \frac{\mathrm{S} 2}{3 \sigma}\right)$ & $\begin{array}{l}\text { 1) } \\
2)\end{array}$ & $\begin{array}{l}\text { Proceso incapaz: } \mathrm{Cp}<1 \\
\text { Proceso aceptable: } 1 \leq \mathrm{Cp} \leq 1,33 \\
\text { Proceso capaz: } \mathrm{Cp} \geq 1,33\end{array}$ \\
\hline $\mathrm{Cp}=\frac{\mathrm{LSE}-\mu}{6 \sigma}, \mathrm{Cp}=\frac{\mu-\mathrm{LIE}}{6 \sigma}$ & $\mathrm{S} 1=\mathrm{LSE}-\overline{\mathrm{X}}$, & $1)$ & $\begin{array}{l}\text { Proceso incapaz: } \mathrm{Cpk}<1 \\
\text { Proceso aceptable: } 1 \leq \mathrm{Cpk} \leq 1,33\end{array}$ \\
\hline
\end{tabular}

LSE: límite superior de especificación, LIE: límite inferior de especificación, $\sigma$ : desviación estándar, $\mu$ : media.

Paso 3, función de pérdida Taguchi: la función de pérdida (Tabla 2) establece una medida financiera del impacto negativo a la sociedad (consumidor y productor) por el desempeño de un producto, cuando se desvía de un valor designado como meta $(\mathrm{m})$. En otras palabras, la característica de calidad de un proceso o producto debe estar cada vez más cerca de su valor ideal, $\mathrm{m}$, y todo lo que se desvíe del ideal es considerado como una pérdida para la sociedad (Gutiérrez-Pulido y de la Vara-Salazar, 2008). 
Tabla 2. Función de pérdida de Taguchi (Gutiérrez-Pulido y de la Vara-Salazar, 2008).

\begin{tabular}{cl}
\hline Fórmulas & \multicolumn{1}{c}{ Variables } \\
\hline & $\mathrm{L}(\mathrm{y})$ : pérdida en términos monetarios. \\
& $\mathrm{y}:$ valor de la característica de calidad. \\
$\mathrm{L}(\mathrm{y})=\mathrm{k}(\mathrm{y}-\mathrm{m})^{2}$ & $\mathrm{~m}$ : valor meta de la característica. \\
$\mathrm{k}=\mathrm{A} / \Delta^{2}$ & $\mathrm{k}:$ coeficiente de costo. \\
& A: pérdida asociada con una unidad en el límite de especificación. \\
& $\Delta:$ tolerancia de la característica. \\
\hline
\end{tabular}

A partir de la definición de la función de pérdida se puede observar que a medida que la característica de calidad (y) se aleja del valor ideal (m), la pérdida aumenta. Tomando esto como base, los esfuerzos de mejora deben estar orientados a reducir la variabilidad de la característica de calidad en torno al valor ideal (m), con lo que la pérdida será cada vez más pequeña.

Etapa 2, propuesta de medidas: luego que quedan identificadas dentro del proceso las causas de mayor variabilidad y el comportamiento actual de estas, es necesario proponer medidas en función de alcanzar oportunidades de mejora en la organización. Dichas medidas serán las obtenidas en la etapa de diagnóstico, a partir de la aplicación de la lista de verificación y el AMFE, luego de ser analizadas, para evitar que haya redundancia y solapamientos entre las mismas.

\section{Resultados y Discusión}

\section{Fase 1: organización del estudio}

En esta fase se procede a conocer la organización y el proceso en el que se desarrolla la investigación y el subsistema de gestión de almacén, para lo cual se definen dos etapas.

Etapa 1, caracterización de la empresa pesquera de Sancti Spíritus: en el año 2000, tras los cambios originados por las reestructuraciones planteadas por el perfeccionamiento empresarial en el ministerio de la industria pesquera, se constituyó la empresa pesquera de Sancti Spíritus. El 20 de mayo del año 2003, comenzó la aplicación del perfeccionamiento empresarial hasta la actualidad, de forma continua e ininterrumpida con avances en su gestión que la distinguen como una de las empresas de su tipo, a nivel de país, con más experiencia liderando las actividades de producción de alevines, captura de especies acuícolas, industrialización, y comercialización de productos derivados de la pesca.

Etapa 2, formación del equipo de trabajo y descripción de los procesos que ocurren en el almacén: para formar el equipo de trabajo se calculó el número de expertos con la Ecuación (1), donde: $\mathrm{i}=0,1$; nivel de confianza= $99 \%$; $\mathrm{p}=0,01$ y $\mathrm{k}=6,6564$; obteniéndose un total de siete expertos. A partir de este análisis, se seleccionaron aquellos con un coeficiente de competencia que estuviera más próximo al valor 1.

Con el apoyo de los expertos, se describió, mediante un diagrama de flujo, el proceso de almacenamiento (Figura 2). Dicho proceso comienza con la solicitud de los productos según el plan a los proveedores; continúa con la recepción a ciega, la facturación en el departamento de economía, la solicitud del cliente y el despacho al mismo, con o sin transportación incluida. 


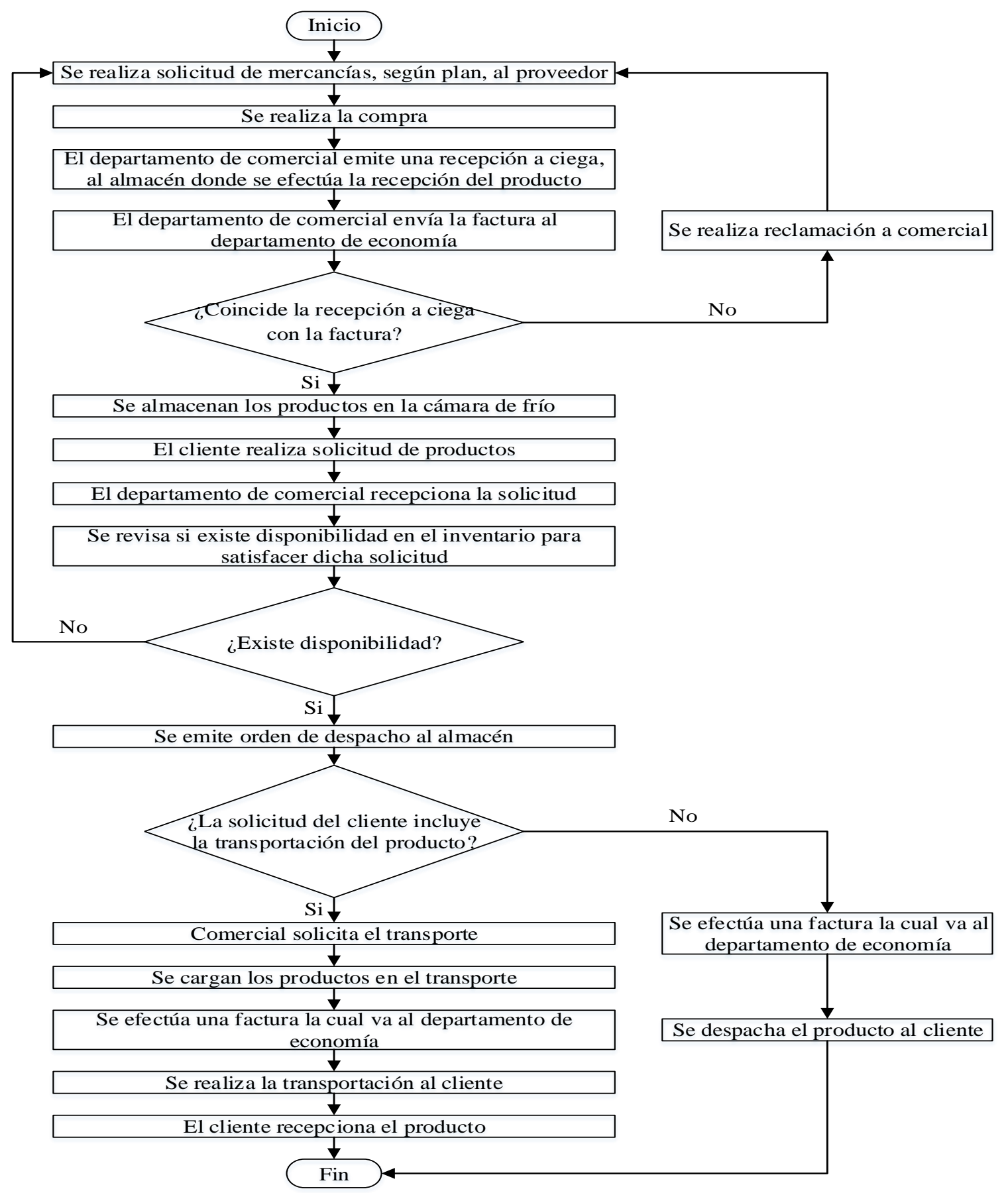

Figura 2. Diagrama de flujo del proceso de almacenamiento de la industria pesquera objeto de estudio.

\section{Fase 2: diagnóstico}

Con el desarrollo de esta fase, se diagnosticó el almacén o cámara de frío y se determinaron los principales riesgos que presenta. 
Etapa 1, diagnóstico del almacén: el diagnóstico de la cámara de frío se realizó mediante una lista de verificación, la cual tiene en cuenta los requisitos de almacenamiento enumerados en la NC 492:2014 (ONN, 2014). Su aplicación arrojó como resultado la existencia de 19 aspectos positivos, para un aproximado del $43 \%$, contra 25 aspectos negativos o deficiencias que representan aproximadamente el $57 \%$. Dentro de estas deficiencias, se encuentran: la temperatura dentro de la cámara no es la adecuada para la conservación de productos pesqueros, insuficiente capacidad de almacenamiento en la cámara de frío y la ausencia de un programa de limpieza y desinfección.

Etapa 2, identificación de los riesgos: para la presente etapa se desarrolló la herramienta conocida como AMFE, específicamente en el proceso de almacenamiento, cuya función es el cuidado y conservación de los productos con el objetivo de preservar sus características originales de calidad. Con esta herramienta se analizaron las principales deficiencias arrojadas por la lista de verificación aplicada en la etapa anterior. Esta proyecta en sus resultados las insuficiencias más importantes a tener en cuenta, es decir, las de mayor NPR, y establece un orden de prioridad además de medidas de tratamiento. Dentro de las de mayor incidencia se encuentran los fallos por temperatura e insuficiente capacidad de almacenamiento. En la Tabla 3, se resumen los principales fallos, sus efectos, sus causas y los niveles de prioridad de riesgo de cada causa.

Tabla 3. Análisis modal de fallos y efectos en el proceso de almacenamiento.

\begin{tabular}{|c|c|c|c|c|}
\hline Fallo & Efectos & Causas & NPR & $\begin{array}{l}\text { Orden de } \\
\text { prioridad }\end{array}$ \\
\hline $\begin{array}{l}\text { Insuficiente capacidad de } \\
\text { almacenamiento en } \\
\text { cámara de frío. }\end{array}$ & $\begin{array}{l}\text { Limitación de la } \\
\text { producción. }\end{array}$ & $\begin{array}{l}\text { Rotura de los difusores de la } \\
\text { segunda cámara de frío, dicha } \\
\text { cámara en desuso. }\end{array}$ & 336 & 3 \\
\hline \multirow{2}{*}{$\begin{array}{l}\text { Temperatura dentro de la } \\
\text { cámara de frío no es la } \\
\text { adecuada para la } \\
\text { conservación de } \\
\text { productos pesqueros. }\end{array}$} & $\begin{array}{l}\text { Reducción de vida útil de } \\
\text { los productos y pérdida de } \\
\text { sus características } \\
\text { originales de calidad } \\
\text { debido al crecimiento } \\
\text { microbiano acelerado. }\end{array}$ & $\begin{array}{l}\text { Obsolescencia tecnológica, } \\
\text { difusores no obtienen temperatura } \\
\text { correcta de }-18{ }^{\circ} \mathrm{C} \text { para productos } \\
\text { pesqueros, existe variaciones de } \\
\text { temperatura donde la mínima } \\
\text { registrada es }-12^{\circ} \mathrm{C} \text {. }\end{array}$ & 480 & 1 \\
\hline & $\begin{array}{l}\text { Los productos no obtienen } \\
\text { temperatura mínima de }-12 \\
{ }^{\circ} \mathrm{C} \text { que alcanza la cámara. }\end{array}$ & $\begin{array}{l}\text { Colocación de los productos no } \\
\text { permiten que el aire circule } \\
\text { libremente alrededor de cada estiba } \\
\text { para mantener la temperatura } \\
\text { central de la masa debido a falta de } \\
\text { estanterías y problemas de } \\
\text { organización. }\end{array}$ & 432 & 2 \\
\hline
\end{tabular}

NPR: nivel de prioridad de riesgo.

\section{Fase 3: control y mejora}

Etapa 1, revisión estadística de las variables y evaluación de las pérdidas: durante todo el mes de abril de 2019 se realizaron mediciones de la temperatura en la cámara de frío, exactamente dos mediciones por día: a las 9:00 am y a las 3:00 pm, lo que implica una muestra de tamaño 2. En la Figura 3 se puede observar el gráfico de control realizado al proceso, donde se evidencia la existencia de valores de temperatura por encima del límite de control superior (LCS:-9,081 ${ }^{\circ} \mathrm{C}$ ) en las muestras $2\left(-8,5^{\circ} \mathrm{C}\right), 13\left(-7,8^{\circ} \mathrm{C}\right)$ y $25\left(-7,9^{\circ} \mathrm{C}\right)$; valores extremadamente bajos para el almacenamiento de productos altamente perecederos, como los productos pesqueros y sus derivados (Xue $e t$ al., 2014). En la Figura 4 se muestra el gráfico de los rangos. 


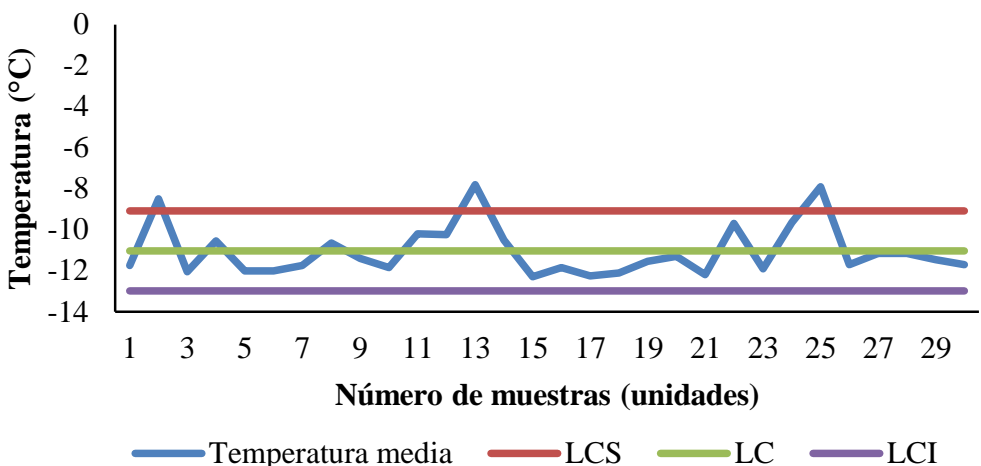

Figura 3. Control de la temperatura en la cámara de frío. LCS: límite de control superior, LC: límite central, LCI: límite de control inferior.

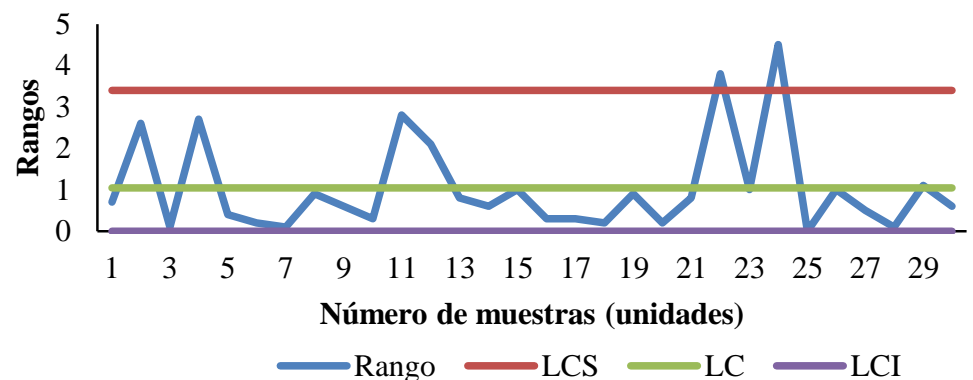

Figura 4. Control de los rangos de la temperatura en la cámara de frío. LCS: límite de control superior, LC: límite central, LCI: límite de control inferior.

En la Figura 5 se muestra el histograma de frecuencia de la temperatura en la cámara de frío, la cual refleja una distribución sesgada a la derecha, se evidencia el desplazamiento paulatino del proceso debido a desgastes o desajustes del sistema de enfriamiento en la cámara, propio de la obsolescencia de estos equipos. Además, se puede apreciar cómo se encuentra dicho proceso completamente fuera de los límites de especificación inferior y superior (Xue et al., 2014).

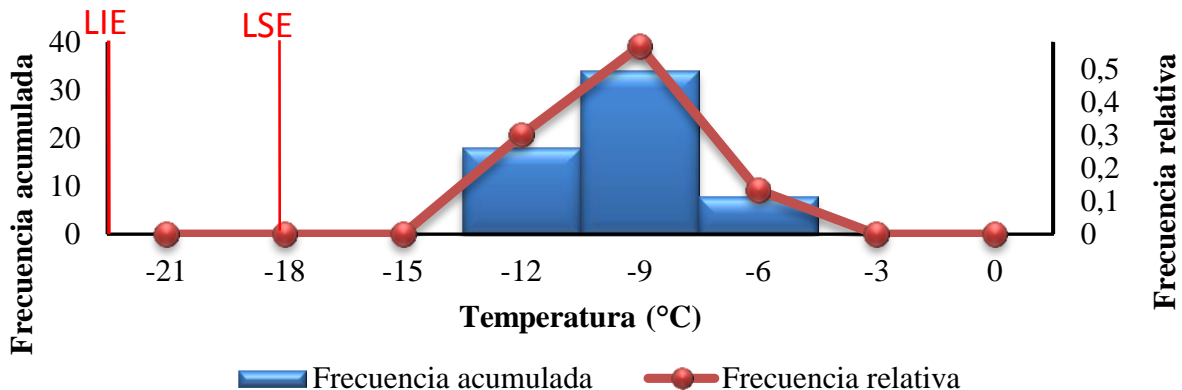

Figura 5. Histograma de frecuencia para la variable temperatura en la cámara de frío. LIE: límite inferior de especificación, LSE: límite superior de especificación.

Con los datos de los límites de especificación, la media y la desviación estándar obtenida, se calculó la capacidad del proceso de almacenamiento, en la cámara de frío (Tabla 4), donde se obtuvo un valor de $\mathrm{Cp}=0,68$, el cual se encuentra muy alejado de las exigencias para procesos existentes con parámetros de seguridad, en los cuales el Cp no debe ser menor a 1,33 (Gutiérrez-Pulido y de la Vara-Salazar, 2009). Por tanto, se llegó a la conclusión de 
que la cámara de frío, bajo las actuales condiciones, no garantiza el cumplimiento de los requisitos de temperatura para el almacenamiento de productos pesqueros.

Tabla 4. Capacidad del proceso de almacenamiento en cámara de frío.

\begin{tabular}{ccccc}
\hline Fórmula & Datos & Cálculo & Límites de evaluación & Conclusión \\
\hline \multirow{2}{*}{$\mathrm{Cp}=\frac{\mathrm{LSE}-\mathrm{LIE}}{6 \sigma}$} & $\begin{array}{c}\sigma=1,4629 \\
\bar{x}=-11,0367\end{array}$ & $\mathrm{Cp}=\frac{-18+24}{6(1,4629)}$ & Proceso incapaz: $\mathrm{Cp}<1$ & \\
$\mathrm{LSE}=-18$ & & Proceso aceptable: $1 \leq \mathrm{Cp} \leq 1,33$ & $0,68<1$ \\
& $\mathrm{LIE}=-24$ & $\mathrm{Cp}=0,6835$ & Proceso capaz: $\mathrm{Cp} \geq 1,33$ & Proceso incapaz \\
& & & & \\
\hline
\end{tabular}

Seguidamente, a partir de los datos recopilados se procedió a analizar el efecto económico que trae consigo la incapacidad del proceso de almacenamiento (Figura 6). Con este fin se utilizó la función de pérdida de Taguchi, obteniéndose la curva que se muestra en la Figura 6.

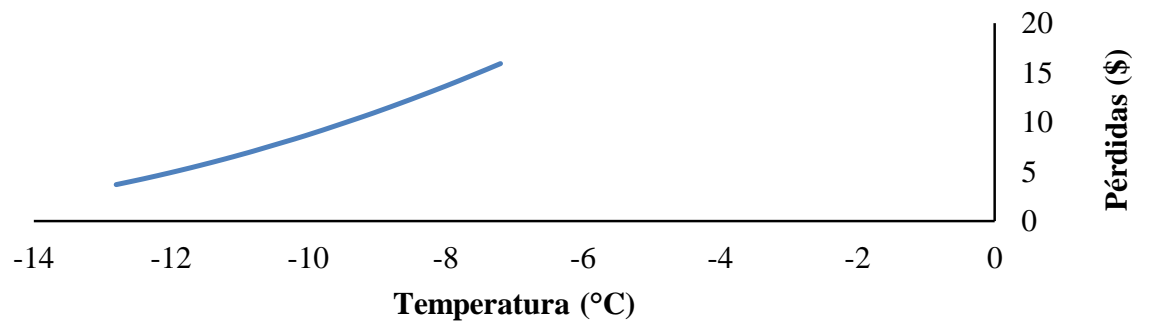

Figura 6. Curva de pérdida, en dinero (\$), en correspondencia con la temperatura de la cámara de frío.

La curva representa para el proceso una pérdida $\mathrm{L}(\mathrm{y})=\$ 13,79$ por día, lo que equivale a una razón del 61,9 $\%$. Esta pérdida se debe a costos extras en los que se incurren por mantener los productos más tiempo en la cámara, por no obtener la temperatura adecuada en las $8 \mathrm{~h}$ que debe estar dentro del almacén, y al deterioro de las características de calidad de uno de los productos con mayor volumen que se almacenan en la cámara de frío como es el picadillo de pescado. Estos $\$ 13,79$ por día representan una pérdida de $\$ 4.965,93$ en la cámara de frío en el período analizado.

Etapa 2, propuestas de medidas: en la presente etapa, se propusieron medidas para la solución de los problemas detectados con la herramienta AMFE en la cámara de frío (Tabla 5). El orden de prioridad en que deben ser aplicadas las medidas está dado por los niveles de prioridad de riesgo, es decir, es prioritario resolver los problemas con los efectos más graves y la probabilidad de ocurrencia más alta.

Tabla 5. Medidas propuestas para la mejora del proceso de almacenamiento en la cámara de frío, según orden de prioridad en AMFE.

NPR Orden de prioridad Medidas

Implementar un sistema de control que permita, mediante herramientas como termómetros e higrómetros, la medición diaria de la temperatura y la $480 \quad 1 \quad$ humedad en la cámara y facilite la toma de medidas correctivas.

Crear un plan de inversión para la adquisición de difusores que logren en la cámara la temperatura de $-18{ }^{\circ} \mathrm{C}$ o inferior a esta.

\begin{tabular}{ccl}
\hline 432 & 2 & $\begin{array}{l}\text { Mejorar la organización y la colocación de las estibas dentro de la cámara } \\
\text { de frío para lograr una mejor circulación del aire alrededor de cada estiba. }\end{array}$ \\
\hline 336 & 3 & $\begin{array}{l}\text { Destinar recursos financieros y materiales a la reparación de la segunda } \\
\text { cámara de frío. }\end{array}$ \\
\hline
\end{tabular}

NPR: nivel de prioridad de riesgo. 


\section{Conclusiones}

Con la aplicación del procedimiento diseñado, se diagnosticó la gestión logística de almacenamiento en la unidad comercializadora de la empresa pesquera de Sancti Spíritus. Con el desarrollo de cada una de las fases y etapas del procedimiento; se identificaron los principales fallos que ocurren en el almacén, los efectos que traen consigo y las causas que los ocasionan. Se midió la variable temperatura y se determinó que no se encontraba en control estadístico. También se calculó la capacidad del proceso de almacenamiento dentro de la cámara de frío y se llegó a la conclusión de que el mismo no es capaz con un $\mathrm{Cp}<1,33$; ocasionado por la obsolescencia del sistema de enfriamiento, por tanto, la cámara de frío no garantiza la temperatura adecuada para la conservación de los productos pesqueros. Por último, se cuantificaron las pérdidas ocasionadas por esta incapacidad del proceso y se proponen medidas para la mejora. A modo de conclusión, el procedimiento se presenta como una herramienta para la mejora continua en la entidad.

\section{Referencias Bibliográficas}

Castillo-Jiménez, D. T., Gómez-Avilés, H. B., de la Cruz-Rivadeneira, O., Rivadeneira-Casanueva, D., LópezConcepción, A., Rodríguez-Fernández, Y. (2021). Diagnóstico del sistema logístico de aprovisionamiento de la industria pesquera en Sancti Spíritus. Revista Técnica de la Facultad de Ingeniería de la Universidad del Zulia, 44(1), 21-28.

Duan, Y., Liu, J. (2019). Optimal dynamic pricing for perishable foods with quality and quantity deteriorating simultaneously under reference price effects. International Journal of Systems Science: Operations \& Logistics, 6(4), 346-355.

FAO. (2012). Pérdidas y desperdicio de alimentos en el mundo. Alcance, causas y prevención. Roma: Food and Agriculture Organization of The United Nations (FAO).

Galindo-López, J., Fraga-Castro, I., Forrellat-Barrios, A., Pelegrín-Morales, E., Cruz-Quintana, Y., Álvarez-Capote, S., Rojas-Maset, G. (2009). Evaluación de diferentes relaciones de proteína animal y vegetal en la dieta de juveniles de camarón blanco Litopenaeus schmitti. Revista Cubana de Investigaciones Pesqueras, 26(1), 1-8.

Gao, A., Tian, Z., Wang, Z., Wennersten, R., Sun, Q. (2017). Comparison between the Technologies for food waste treatment. Energy Procedia, 105(1), 3915-3921.

Gutiérrez-Pulido, H., de la Vara-Salazar, R. (2008). Análisis y diseño de experimientos. México: McGraw-Hill.

Gutiérrez-Pulido, H., de la Vara-Salazar, R. (2009). Control estadístico de calidad y seis sigma. México: McGrawHill.

James, P. (1997). La gestión de la calidad total: un texto introductorio. Madrid, España: Prentice-Hall.

Kaufmann, L., Gaeckler, J. (2015). A structured review of partial least squares in supply chain management research. Purchasing and Supply Management, 21(1), 259-272.

Koldborg, T., Nielsen, J., Larsen, E., Clausen, J. (2013). The fish industry. Toward supply chain modeling. Journal of Aquatic Food Product Technology, 19(3), 214-226.

Lemma, Y., Kitaw, D., Gatew, G. (2014). Loss in perishable food supply chain: an optimization approach literature review. International Journal of Scientific \& Engineering Research, 16(5), 302-308.

ONN NC 49:1981. (1981). Control de la calidad. Métodos de expertos. La Habana, Cuba: Oficina Nacional de Normalización (ONN).

ONN NC 492:2014. (2014). Almacenamiento de alimentos. Requisitos sanitarios generales. La Habana, Cuba. Oficina Nacional de Normalización (ONN). 
Pulido, J. (2014). Gestión de la cadena de suministros. El último secreto. Caracas, Venezuela: Torino.

Sánchez, V., Hasbleidy, Z. (2014). Modelos y configuraciones de cadenas de suministro en productos perecederos. Revista Ingenieria y Desarrollo, 32(1), 138-154.

Xue, M., Zhang, J., Tang, W. (2014). Optimal temperature control for quality of perishable foods. ISA transactions, $53(2), 542-546$. 


\section{REVISTA TECNICA}

DE LA FACULTAD DE INGENIERIA

UNIVERSIDAD DEL ZULIA

Vol. 44. $\mathbf{N}^{\circ} 3$, Septiembre - Diciembre, 2021

Esta revista fue editada en formato digital y publicada en Agosto 2021, por el Fondo Editorial Serbiluz, Universidad del Zulia. Maracaibo-Venezuela

www.luz.edu.ve

www.serbi.luz.edu.ve

www.produccioncientificaluz.org 